Pacific Journal of Mathematics

NUCLEAR SPACES, SCHAUDER BASES, AND CHOQUE 


\title{
NUCLEAR SPACES, SCHAUDER BASES, AND CHOQUET SIMPLEXES
}

\author{
A. J. LAZAR AND J. R. RETHERForD
}

We prove that a Frechet space $E$ with a Schauder basis is nuclear if and only if every bounded subset of $E$ is contained in a bounded Choquet simplex. We then show how this theorem relates to the basis problem for nuclear Frechet spaces and to the classical Banach-Dieudonné theorem.

In the class of all infinite dimensional Banach spaces the Hilbert spaces (and their isomorphs) seem to be the natural generalization of the finite dimensional normed linear spaces. Indeed many authors have characterized Hilbert spaces by extending results from Euclidean geometry (see [4, Chap. VII, $\S 3$ p. 115-121] for an excellent discussion).

However, in the class of all infinite dimensional Fréchet spaces (complete, metrizable, locally convex) this is no longer the case. It now appears that the nuclear spaces of A. Grothendieck [9], [26] are the proper generalizations of the finite dimensional spaces.

Indeed, Grothendieck [9] has shown that a Frechet space $E$ is nuclear if and only if $E$ has the Dvoretzky-Rogers property: every unsonditionally convergent series in $E$ is absolutely convergent.

This property is known to characterize the finite dimensional normed linear spaces in the class of all normed linear spaces [7].

Also, it is well known that nuclear spaces can be nicely approximated by finite dimensional spaces (Kolmogorov diameters, diametric and approximative dimensions) see [1], [22] and [26]. Moreover, the Dynin-Mitiagin theorem [8] and a recent result of Wojtynski [29] asserts that a Fréchet space $E$ with a Schauder basis is nuclear if and only if every basis of $E$ is absolute. Since pelczynski and Singer [23] have shown that every infinite dimensional Banach space with a basis has a conditional basis, the above result can be viewed as a generalization to the class of Fréchet spaces with bases of a wellknown property of finite dimensional spaces.

Aside from these characterizations and a few related results, there seems to be few instances where nuclear spaces have been characterized by some property common to finite dimensional normed linear spaces. The purpose of this paper is to present such a characterization.

It is obvious that a bounded set in a finite dimensional normed linear space lies in a bounded simplex. Our goal is to prove

Theorem 1. A Fréchst space $E$ with a Schauder basis is nuslear if and only if every bounded subset of $E$ is contained in a bounded 
Choquet simplex. (Moreover, this simplex can be taken to be compact).

Part of this result, without the basis requirement, has appeared in [18].

2. Terminology. All linear-topological prerequisites can be found in Köthe [15] and Day [4]. Throughout this paper $E$ denotes a real Fréchet space. We will use the word neighborhood to mean "closed convex circled neighborhood"'.

If $U$ is a neighborhood of the origin in $E$ with Minkowski functional $\rho_{U}$ then by $E_{U}$ we denote the quotient space $E / \rho_{U}^{-1}(0)$, on which $\rho_{U}$ is a norm. The space $E$ is nuclear [26] if for each neighborhood $U$ of 0 in $E$ there is a neighborhood of $0, V$, absorbed by $U$, such that the canonical map

$$
I_{V U}: E_{V} \rightarrow E_{U}
$$

is nuclear, i.e.

$$
I_{V U}(x)=\sum_{n=1}^{\infty} \lambda_{n}\left\langle x, f_{n}\right\rangle o_{n}, \quad x \in E_{V}
$$

where $\sum_{n=1}^{\infty}\left|\lambda_{n}\right|<+\infty,\left(f_{n}\right) \subset E_{V}^{\prime}$ is equicontinuous and $\left(\rho_{n}\right) \subset E_{U}$ is bounded.

(For equivalent ways to define nuclearity see [26].) We will use conv $A$ and $\Gamma(A)$ to denote, respectively, the convex hull and convex circled hull of a set $A$ in $E$. As usual, $\bar{A}$ denotes the closure of $A$.

A sequence $\left(x_{n}\right)$ in $E$ is topologically free if no $x_{n}$ is in the closed linear span of $\left(x_{m}: m \neq \equiv n\right)$; equivalently, $\left(x_{n}\right)$ is topologically free if there are functionals $\left(f_{n}\right)$ in $E^{\prime}$ such that

$$
f_{n}\left(x_{m}\right)=\delta_{n m} \text {, the Kronecker delta. }
$$

(See [5] for a discussion of topologically free sequences in locally convex spaces.) A topologically free sequence $\left(x_{n}\right)$ with associated functionals $\left(f_{n}\right)$ is a Schauder basis for $E$ if for each $x \in E$,

$$
x=\sum_{n=1}^{\infty} f_{n}(x) x_{n}
$$

convergence in the topology of $E$.

If $A$ is a set of positive sequences $a=\left(a_{n}\right)$ (i.e., all $a_{n} \geqq 0$ ) satisfying

(i) for each $n$ there is an $a \in A$ such that $a_{n} \neq 0$; and,

(ii) for $a^{1}, \cdots, a^{n} \in A$ there is an $a \in A$ and $M>0$ such that

$$
\begin{gathered}
a_{n}^{i} \leqq M a_{n} \text { for all } i \text { and } n, \text { then } \\
\lambda(A)=\left\{x=\left(x_{n}\right): \rho_{a}(x)=\sum_{n=1}^{\infty} a_{n}\left|x_{n}\right|<+\infty \text { for all } a \in A\right\}
\end{gathered}
$$


with the topology generated by the seminorms $\left\{\rho_{a}: a \in A\right\}$ is called a Köthe sequence space. It follows from the Dynin-Mitiagin theorem above that a nuclear Fréchet space $E$ with a Schauder basis $\left(x_{n}\right)$ is isomorphic to the Köthe sequence space $\lambda(A), A=\left\{\left|x_{n}\right|_{m}\right\}$, where $|\cdot|_{1} \leqq|\cdot|_{2} \leqq \cdots$ is an increasing family of seminorms generating the topology of $E$. Thus, nuclear Fréchet Köthe sequence spaces coincide with the class of nuclear Fréchet spaces with bases. (The above discussion is the main theme of the work [1]; for general relations between bases and sequence spaces see [28]).

In general, $\lambda=\lambda(A)$ is nuclear if and only if for each $x \in \lambda$ there is a $y \in \lambda$ and $t \in l_{1}$ with $x_{n} \leqq t_{n} y_{n}$ for each $n$. This property is known as the Grothendieck-Pietsch criterion [14].

Grothendieck has conjectured that every nuclear Fréchet space has a Schauder basis. Our Theorem 2 below sheds some new light on this (still unsolved) conjecture.

Finally we need the notion of a Choquet simplex.

Let $S$ be a convex subset of $E$. Passing to $E \times R, R$ the scalar field, if necessary, we may suppose that $S$ lies in a hyperplane of $E$ which misses the origin. The set $S$ is a Chsqust simplex [3], [25] if the cone

$$
C=\{\alpha s: \alpha \geqq 0, s \in S\}
$$

generated by $S$ induces a lattice order in $C-C$. Choquet has observed that in finite dimensional spaces a Choquet simplex coincides with the usual notion of simplex. (A word of caution is necessary. A Choquet simplex $S$ may not, strictly speaking, generate a cone in $E$, e.g. if the origin is in the core of $S$. More precisely, $S$ is a Choquet simplex if $S$ is affinely homeomorphic to a set $S^{\prime}$ with the above properties. In particular, a translate of a Choquet simplex is again a Choquent simplex.)

3. Three lemmas. In this section we develop the machinery used to prove Theorems 1 and 2. Lemma 1, purely technical in nature, appears in [18]. We reproduce it here for completeness. Lemmas 2 and 3 are of independent interest.

Lemma 1. Let $(T, \Sigma, \mu)$ be a measure space and $\left(g_{n}\right)_{n=0}^{\infty}$ a sequence in $L_{1}(T, \Sigma, \mu)$. Suppose there is a constant $M$ such that $g_{0} \geqq 0, g_{0}+$ $\sum_{n \in \sigma} g_{n} \geqq 0$ and $\int_{T} g_{0} d \mu+\sum_{n \in \sigma} \int_{T} g_{n} d \mu \leqq M$ for any finite set $\sigma$ of positive integers. Then

$$
\sum_{n=0}^{\infty} \int_{T}\left|g_{n}\right| d \mu<+\infty
$$


Proof. Since $L_{1}(T, \Sigma, \mu)$ is weakly complete and

$$
\left\|\sum_{n \in \sigma} g_{n}\right\| \leqq M+\left\|g_{0}\right\|
$$

it follows that $\sum_{n=0}^{\infty} g_{n}$ is unconditionally convergent [4, p. 60], [21], say to $g$. For any positive integer $m$ let $\left(E_{i}\right)_{i=1}^{p(m)}$ be a partition of $T$ into disjoint measurable sets such that each $g_{n}, 1 \leqq n \leqq m$ has constant sign on $E_{i}, 1 \leqq i \leqq p(m)$.

For fixed $i$ let

$$
P_{i}=\left\{n: g_{n} \mid E_{i} \geqq 0,1 \leqq n \leqq m\right\},
$$

and

$$
Q_{i}=\{1,2, \cdots, m\}-P_{i} .
$$

Then

$$
\sum_{n \in P_{i}} \int_{E_{i}}\left|g_{n}\right| d \mu=\int_{E_{i}}\left(\sum_{n \in P_{i}} g_{n}\right) d \mu \leqq \int_{E_{i}} g d \mu
$$

and

$$
\sum_{n \in Q_{i}} \int_{E_{i}}\left|g_{n}\right| d \mu=-\int_{E_{i}}\left(\sum_{n \in Q_{i}} g_{n}\right) d \mu \leqq \int_{E_{i}} g_{0} d \mu
$$

Thus

$$
\sum_{n=1}^{m} \int_{E_{i}}\left|g_{n}\right| d \mu \leqq \int_{E_{i}}\left(g_{0}+g\right) d \mu
$$

and so,

$$
\sum_{n=1}^{m} \int_{T}\left|g_{n}\right| d \mu \leqq \int_{T}\left(g_{0}+g\right) d \mu<+\infty .
$$

The next lemma is the main tool needed in the proofs of Theorems 1 and 2.

Lemma 2. Let $\left(y_{n}\right)$ be a topologically free null sequence in a locally convex space. If $K=\overline{\mathrm{conv}}\left(y_{n}\right)$ is compact, then $K$ is a Choquet simplex.

Proof. By Milman's theorem [15, p. 332] the set of extreme points of $K$, ext $K$, is included in $\left\{y_{n}: n \geqq 1\right\} \cup\{0\}$. By the ChoquetMeyer uniqueness theorem [25, p. 66] we must show that every point $k \in K$ admits a unique maximal (in Choquet's order) representing measure. The set ext $K$ is closed since $\left(y_{n}\right)$ is a null sequence and so the maximal measures on $K$ are exactly those measures supported by ext $K[25$, p. 27, p. 30 and Prop. 9.3, p. 69], i.e. they are atomic 
measures supported by $\left\{y_{n}: n \geqq 1\right\} \cup\{0\}$. Let $\mu$ and $\nu$ be two maximal measures representing $k \in K$. Let $\mu\left(\left\{y_{n}\right\}\right)=\mu_{n}, \mu(\{0\})=\mu_{0}$ and similarly for $\nu_{n}$ and $\nu_{0}$. Then $\mu_{n}, \nu_{n} \geqq 0$ for $n=0,1,2, \cdots$,

and

$$
(+) \quad \sum_{n=0}^{\infty} \mu_{n}=\sum_{n=0}^{\infty} \nu_{n}=1 \text {, }
$$

$$
k=\sum_{n=1}^{\infty} \mu_{n} y_{n}=\sum_{n=1}^{\infty} \nu_{n} y_{n}
$$

Let $\left(f_{n}\right)$ be the functionals associated with $\left(y_{n}\right)$. Then $f_{n}(k)=\mu_{n}=\nu_{n}$ and from $(+)$ it follows that $\mu_{0}=\nu_{0}$, i.e. $\mu=\nu$ and $K$ is a Choquet simplex.

It is very likely that the conclusion of Lemma 2 holds without the assumption that $K$ is compact. In the case of a Fréchet space, our interest here, of course $K$ is compact. Thus we have not checked the general result.

For our final lemma we need the notation of the normal hull of a set of sequences. If $M$ is a set of real sequences $b=\left(b_{n}\right)$, the normal hull of $M$ is the set of all real sequences $y=\left(y_{n}\right)$ with $\left|y_{n}\right| \leqq\left|b_{n}\right|$ for all $n$ and some $b \in M$.

Köthe [14] has shown that in a nuclear sequence space $\lambda$, each bounded set $B$ is contained in the normal hull of a single element $\rho=\left(\rho_{n}\right)$.

Geometrically Lemma 3 says that in a nuclear sequence space a "parallelopiped" can be placed in a simplex.

LEMMA 3. Let $\lambda$ be a nuclear Köthe sequence space and let $\rho=$ $\left(\rho_{n}\right) \in \lambda, \rho_{n} \geqq 0$ for each $n$. Then $B=\left\{x \in \lambda:\left|x_{n}\right| \leqq \rho_{n}\right\}$ is contained in a compact Choquet simplex.

Proof. Considering $B+\rho$ instead of $B$ we may assume $B=$ $\left\{x \in \lambda: 0 \leqq x_{n} \leqq \rho_{n}\right\}$. By the Grothendieck-Pietsch criterion there is a $\sigma=\left(\sigma_{n}\right) \in \lambda$ and $\left(t_{n}\right) \in l_{1}$ such that $\rho_{n} \leqq t_{n} \sigma_{n}$. Dividing by a constant if necessary we can assume that $\Sigma t_{n} \leqq 1, t_{n}>0$. Also we can suppose that no $\sigma_{n}$ is zero. If $\left(e_{n}\right)$ is the unit vector basis of $\lambda$ then $\left(\sigma_{n} e_{n}\right)$ is a topologically free null sequence. Thus if $x \in B, x=\Sigma x_{n} e_{n}=$ $\Sigma t_{n}\left(\sigma_{n} t_{n}\right)^{-1} x_{n} \sigma_{n} e_{n}$ and $\Sigma\left|t_{n}\left(\sigma_{n} t_{n}\right)^{-1} x_{n}\right| \leqq \Sigma t_{n} \leqq 1$ i.e. $B \subset \overline{\Gamma\left(\sigma_{n} e_{n}\right)}$. By the Dynin-Mitiagin theorem $\Sigma \sigma_{n} e_{n}$ is absolutely convergent to $\sigma$. Let $S=\overline{\mathrm{conv}}\left(2 \sigma_{n} e_{n}\right)$. By Lemma $2, S$ and hence any translate of $S$, is a compact Choquet simplex. Thus $B+\sigma \subset \overline{\Gamma\left(\sigma_{n} e_{n}\right)}+\sigma \subset S$, i.e..

$$
B \subset S-\sigma
$$

4. Proof of theorem 1. We first suppose that $E$ is a nuclear Fréchet space with a basis. If $B \subset E$ is bounded then by the result 
of Köthe above $B$ is contained in the normal hull of some $\rho=\left(\rho_{n}\right) \in \lambda$, the sequence space representation of $E$. By Lemma $3, B$ is contained in a compact Choquet simplex.

Now suppose each bounded subset of $E$ is contained in a bounded Choquet simplex. Let $\sum_{n=1}^{\infty} x_{n}$ be an unconditionally convergent series in $E$ and let $A=\left\{\sum_{n \in \sigma} x_{n}: \sigma\right.$ finite $\} \cup\{0\}$. It is well known that $A$ is precompact, hence by hypothesis, contained in a bounded Choquet simplex $B$. Consider in $E \times R$ the cone $C$ generated by $B \times\{1\}$. Then $F=C-C$ is a linear lattice with the order induced by $C$. The Minkowski functional of $\Gamma(B \times\{1\})$ is a norm on $F$. This norm and the order are related by the following two properties:

(i) if $x \wedge y=0$ then $\|x+y\|=\|x-y\|$; and

(ii) if $x \geqq 0, y \geqq 0$ then $\|x+y\|=\|x\|+\|y\|$.

It follows from the Kakutani theorem [16], [4, p. 98-100] that the completion of $F$ is an abstract $L$-space (with order determined by $\bar{C}$ ).

Let $P$ be the restriction to $F$ of the natural projection from $E \times R$ onto $E$. Since $B$ is bounded, $P$ is cotinuous from $F$ to $E$. Let $y_{n}=\left(x_{n}, 1\right), n=0,1,2, \cdots \quad$ where $x_{0}=0$. Clearly $y_{0} \in B \times\{1\}$ and $y_{0}+\sum_{n \in \sigma}\left(y_{n}-y_{0}\right) \in B \times\{1\}$ for each finite set of positive integers $\sigma$. But $B \times\{1\}$ is part of the positive face of the unit ball in an abstract $L$-space. By Lemma 1 it follows that $\sum_{n=1}^{\infty}\left\|y_{n}-y_{0}\right\|<+\infty$. Since $P y_{n}=x_{n}$ it follows from the continuity of $P$ that $\Sigma x_{n}$ is absolutely convergent, i.e. $E$ has the Dvoretzky-Rogers property. By the result of Grothendieck quoted in the introduction, $E$ is nuclear.

5. Cones and the existence of bases. In any separable Fréchet space $E$ (in particular a nuclear Fréchet space) there is a topologically free null sequence $\left(z_{n}\right)$ whose closed linear span is $E$, i.e. $\left(z_{n}\right)$ is fundamental. (This result is due essentially to Markuchevitch [19]; see also Klee [11]). Thus $S=\overline{\mathrm{conv}}\left(z_{n}\right)$ is, by Lemma 2, a Choquet simplex. It is not hard to see that $C=\{\alpha s: \alpha \geqq 0, s \in S\}$ is always a proper cone. There are important cases in which $\bar{C}$ is not only a cone but makes $\bar{C}-\bar{C}$ a vector lattice (see [24] for terminology). We will call a simplex $S$ a VL-simplex if $\bar{C}$ is a cone making $\bar{C}-\bar{C}$ a vector lattice.

Let $\left(x_{n}\right)$ be an unconditional basis for $E$ with coefficient functionals $\left(f_{n}\right)$ and let $K=\left\{x \in E: f_{n}(x) \geqq 0\right.$ for all $\left.n\right\}$. Then $K$ is called the basis cone of $\left(x_{n}\right)$.

Ceitlin [2] has shown that $K$ is normal (for any two nets $\left(x_{\beta}\right),\left(y_{\beta}\right)$ in $E$ with $0 \leqq x_{\beta} \leqq y_{\beta}$, if $\lim _{\beta} x_{\beta}=0$ then $\lim x_{\beta}=0$ ) and $K-K=E$. Ceitlin observes that in this situation the induced lattice operations are continuous. The work of Ceitlin above makes it easy to prove the following result.

Proposition 1. If $\left(x_{n}\right)$ is an unconditional basis in a Fréchet 
space $E$ with $\lim _{n} x_{n}=0$ then $S=\overline{\mathrm{conv}}\left(x_{n}\right)$ is a VL-simplex.

Proof. By Lemma 2 and the above remarks we need only observe that $\bar{C}=K$ where $C=\{\alpha s: \alpha \geqq 0, s \in S\}$ and $K$ is the basis one of $\left(x_{n}\right)$. The inclusion $\bar{C} \subset K$ is obvious and since any finite sum $\sum_{i=1}^{n} a_{\imath} x_{i}$, $a_{i} \geqq 0$ is in $C, K \subset \bar{C}$.

Our next theorem shows the difficulties in trying to remove the basis hypothesis in Theorem 1 .

Theorem 2. Let $E$ be a nuclear Fréchet space. The following are equivalent:

(a) $E$ has a Schauder basis;

(b) each bounded subset of $E$ is contained in a translate of a bounded VL-simplex; and

(c) there is a fundamental bounded set containing 0 and contained in a translate of a bounded VL-simplex.

Proof. (a) $\rightarrow\left(\right.$ b): Let $\left(e_{n}\right)$ be a basis for $E, B$ a bounded subset of $E$ and let $\rho=\left(\rho_{n}\right), \sigma=\left(\sigma_{n}\right)$ have the meaning of Lemma 3 .

Then by Lemma $3, B \subset-\rho+S$ where $S=\overline{\operatorname{conv}}\left(2 \sigma_{n} e_{n}\right)$. Since we may assume that each $\sigma_{n} \neq 0\left(2 \sigma_{n} e_{n}\right)$ is, by the Dynin-Mitiagin theorem, an unconditional basis for $E$. Also $\left(2 \sigma_{n} e_{n}\right)$ is a null sequence. By Proposition $1, S$ is a $V L$-simplex.

(b) $\rightarrow$ (c) is obvious from [19] since $E$ is separable.

(c) $\rightarrow$ (a). Let $B$ be the fundamental bounded set of (c). By hypothesis there is a $\rho \in E$ and a $V L$-simplex $S$ such that $B-\rho \subset S$. If $C$ is the cone generated by $S$ then, since $0 \in B$

$$
B \subset B-B=(B-\rho)-(B-\rho) \subset \bar{C}-\bar{C} \text { i.e. }
$$

$\bar{C}-\bar{C}$ is dense in $E$. Since $E$ is nuclear and $S$ is a $V L$-simplex $\bar{C}-\bar{C}$ is a nuclear vector lattice and so [13] the lattice operations can be extended to $E$ in such a way that $E$ is a nuclear vector lattice. However, from the generalized Grinblyum $K$-condition [20] and the results of Kōmura-Koshi [13] it follows that every Fréchet nuclear vector lattice has a basis. (See [1] for a complete discussion of the Kōmura-Koshi result and its relation to basis theory.)

6. The strong Banach-Dieudonne property. Let us recall the Banach-Dieudonné theorem for Fréchet spaces: every precompact subset of $E$ is contained in the closed convex circled hull of a null sequence (see e.g. [15, p. 273]).

The following proposition allows one to strengthen this result. 
Proposition 2. Let $\left(x_{n}\right)$ be a null sequence in a locally convex space $F$. Then there is either a linearly independent null sequence or a finitely nonzero sequence $\left(z_{n}\right)$ in $F$ such that $\overline{\operatorname{conv}}\left(x_{n}\right) \subset \overline{\operatorname{conv}}\left(z_{n}\right)$.

Proof. If there is no infinite dimensional bounded set in $F$ then $\overline{\mathrm{conv}}\left(x_{n}\right)$ is bounded and finite dimensional and the assertion is obvious. Thus suppose $\left(y_{n}\right)$ is a bounded linearly independent sequence in $F$. Construct an infinite array in the following manner: If $x_{1}, \cdots, x_{i}$ are linearly independent and $x_{i+1}=\sum_{j=1}^{i} \lambda_{j} x_{j}$ put $x_{j}, 1 \leqq j \leqq i$ in the first place of the $j$-th row. Choose $n_{1}$ such that $n_{1}^{-1} y_{n_{1}}+x_{i+1}$ is linearly independent from the elements in the array and put this new element in the first place of the $i+1$-row. If $x_{i+2}$ is linearly independent from the elements of the array put it in the first place of the $i+2$-row. If not, put in this place $n_{2}^{-1} y_{n_{2}}+x_{i+2}$ where $n_{2}>n_{1}$ and $n_{2}^{-1} y_{n_{2}}+x_{i+2}$ is linearly independent from the elements of the array. Next choose $n_{3}>n_{2}$ such that $n_{2}^{-1} y_{n_{3}}+x_{i+1}$ is linearly independent from the elements of the array and put it in the second place of the $i+1$-row. The first element of the $i+3$-row will be $x_{i+3}$ or of the form $n_{4}^{-1} y_{n_{4}}+x_{i+3}$ (where $n_{4}>n_{3}$ ) depending on whether $x_{i+3}$ is linearly independent or not from the elements of the array. Next put $n_{5}^{-1} y_{n_{5}}+x_{i+1}, n_{\mathrm{d}}^{-1} y_{n_{6}}+x_{i+2}$ with $n_{6}>n_{5}>n_{4}$ in the third place of the $i+1$-row and the second place of the $i+2$-row (if the row does not begin with $x_{i+2}$ ), where these elements are chosen to be linearly independent from the array. Continue the process and enumerate the array diagonally to obtain the sequence $\left(z_{n}\right)$. Then $\left(z_{n}\right)$ is a null sequence and $\left(x_{n}\right) \subset \overline{\mathrm{conv}}\left(z_{n}\right)$.

In particular if $E$ is metrizable, Proposition 2 and the BanachDieudonné theorem show that every precompact subset of $E$ is contained in the closed convex circled hull of a linearly independent null sequence.

This motivates the following definition. We say that a locally convex space $F$ has the strong Banach-Dieudonno property if each bounded subset of $F$ is contained in the closed convex circled hull of a topologically free null sequence.

REMARK 1. If $F$ is barrelled and has the strong Banach-Dieudonné property then $F$ is a Montel [15] space. Indeed, in this case, bounded sets are necessarily precompact. In particular, if a Banach space $E$ has the strong Banach-Dieudonné property then $E$ is finite dimensional.

If one replaces "null sequence" in the above definition with "absolutely convergent sequence" then the proof of Lemma 2 and [26] show that a space $F$ with this property must be nuclear.

We do not know if a Fréchet space $E$ with the strong Banach- 
Dieudonné property is necessarily nuclear. Lemma 3 shows that a nuclear Fréchet space with a Schauder basis $\left(e_{n}\right)$ has the strong Banach-Dieudonné property. Indeed if

$$
B=\left\{x \in \lambda:\left|x_{n}\right| \leqq \rho_{n}\right\}
$$

then by the proof of Lemma 3

$$
A=\left\{y \in \lambda: 0 \leqq y_{n} \leqq 2 \rho_{n}\right\} \subset \overline{\Gamma\left(\sigma_{n} e_{n}\right)} .
$$

Also,

$$
-A \subset \overline{\Gamma\left(\sigma_{n} e_{n}\right)} \text { and } B \subset \overline{\operatorname{conv}}(A \cup-A)
$$

since $\Sigma x_{n} e_{n}$ is we conclude with two examples.

ExAmPle 1. A nuclear space $F$ which lacks the strong BanachDieudonné property.

Let $F$ be $l_{1}$ with the $\sigma\left(l_{1}, l_{\infty}\right)$-topology. Since $F$ has a neighborhood basis $\{U\}$ such that for each $U \in\{U\}, F_{U}$ is finite dimensional, $F$ is nuclear. By Shur's lemma sequential convergence in $F$ is norm convergence in $l_{1}$. If $B$ is the unit ball of $l_{1}$ there is no null sequence $\left(y_{n}\right)$ in $F$ with $B \subset \Gamma\left(y_{n}\right)$.

EXAMPle 2. A locally convex space $F$ with the strong BanachDieudonné property which is not nuclear.

Let $F$ be $l_{1}$ with the bounded weak*-topology (i.e. the topology of uniform convergence on null sequences in $c_{0}$ ). Since a functional $f$ is $b w^{*}$-continuous if and only if it is $w^{*}$-continuous [6], a bounded subset of $F$ is $w^{*}$-bounded, hence norm bounded. Thus to show that $F$ has the strong Banach-Dieudonné property it suffices to show that the unit ball of $l_{1}$ is contained in the closed convex circled hull of a topologically free $F$-null sequence. Let $\left(e_{n}\right)$ be the unit vector basis of $l_{1}$. Clearly $B \subset \overline{\Gamma\left(e_{n}\right)}$ and $\left(e_{n}\right)$ is topologically free. Also $w^{*}-\lim e_{n}=0$ and so by [4, p. 42] $\left(e_{n}\right)$ is $F$-null.

To see that $F$ is not nuclear it suffices [26] to produce a series $\Sigma x_{n}$ in $F$ which is unconditionally but not absolutely, convergent.

By the proof of the Dvoretzky-Rogers theorem [7], [4] there is a sequence $\left(x_{n}\right)$ in $l_{1}$ such that $\Sigma x_{n}$ is unconditionally convergent (in norm) and $\left\|x_{n}\right\|=1 / n$. For each $n$ let $y_{n} \in c_{0},\left\|y_{n}\right\|=1$ and $x_{n}\left(y_{n}\right)>1 / 2 n$. Let $y$ be the null sequence $\left((1 / \ln m) y_{m}\right)$. Then

$$
\rho_{y}\left(x_{n}\right)=\sup _{m}\left|\frac{1}{\ln m} x_{n}\left(y_{m}\right)\right| \geqq \frac{1}{2 n \ln n} .
$$


Thus $\Sigma \rho_{y}\left(x_{n}\right)$ diverges. But $\rho_{y}$ is a continuous seminorm on $F$ and so $\Sigma x_{n}$ is not absolutely convergent in $F$.

\section{BIBLIOGRAPHY}

1. C. Bessaga and J. R. Retherford, Lectures on Nuclear Spaces, Lecture Notes LSU, 1967 (Manuscript to appear).

2. Ja. M. Ceitlin, Unconditionality of a basis and partial order, (Russian) Izv. Vyss. Ucebn. Zaued. Matematika 1966 No. 2 (51) 98-104.

3. G. Choquet, Existence et unicité de representations intégrales au moyen des points extremaux dans les cônes convexes, Séminaire Bourbaki (Dec. 1956), 139, 15 pp.

4. M. M. Day, Normed linear spaces, Springer-Verlag, Berlin, 1962.

5. J. Dieudonné, On biorthogonal systems, Michigan Math. J., 2 (1953/1954), 7-20.

6. N. Dunford and J. T. Schwartz, Linear Operator, Part 1. New York: Interscience Publ. Inc. 1958.

7. A. Dvoretzky and C. A. Rogers, Absolute and unconditional convergence in normed linear spaces, Proc. Nat. Acad. Sci., (USA) Vol. 36, No. 3 (1950), 192-197.

8. A. S. Dynin and B. S. Mitiagin, Criterion for nuclearity in terms of approximative dimension, Bull. Acad. Polon. Sci., 8 (1960), 535-540.

9. A. Grothendieck, Produits tensoriels topologiques et espaces nucléaires, Memoirs of Amer. Math. Soc., (1955), No. 16.

10. - Résumé des résultats essentiels dans la théorie des produits tensoriels topologiques, Bol. Soc. Matem. Sao Paulo 8 (1956), 1-79.

11. V. Klee, On the Borelian and projective types of linear subspaces, Math. Scand., 6 (1958), 189-199.

12. Y. Kōmura and T. Kōmura, Über de eibettung der nuklearern Räume in $(s)^{A}$, Math. Annalean, 162 (1966), 284-288.

13. Y. Kōmura and S. Koshi, Nuclear Vector lattices, Math. Annalen, 163 (1966), $105-110$.

14. G. Köthe, Uber nucleare folgenraume, Studia Math., 31 (1968), 267-271.

15. - Topological Vector Spaces I, Springer-Verlag Berlin 1969 (Trans. of Topologische linear Raume 1960).

16. S. Kakutani, Concrete representation of abstract $(L)$-spaces and the mean ergodic theorem, Ann. of Math., (2) 42 (1941), 523-537.

17. - Concrete representation of abstract (M)-spaces, Ann. of Math., (2) 42 (1941), 994-1024.

18. A. Lazar, Sections and subsets of simplexes, Pacific J. Math., 33 (1970), 337-345.

19. A. Markouchevitch, Sur les bases (au sens large) dans les espaces linéaires, C. R. (Doklady) Acad. Sci. URSS (N.S.) 41 (1943), 227-229.

20. C. W. McArthur and J. R. Retherford, Some remarks on bases in linear topological spaces, Math. Ann., 247 (1966), 38-41.

21. - Some Applications of an inequality in locally convex spaces, Trans. Amer. Math. Soc., 137 (1969), 115-123.

22. B. S. Mitiagin, Approximative dimension and bases in nuclear spaces (Russian) USP. Mat. Nauk 16, 4 (100) (1961), 63-132 (English translation: Russian Math. Surveys 16 (1961) 9, 59-127.)

23. A. Pelczynski and I. Singer, On non-equivalent bases and conditional bases in Banach spaces, Studia. Matn., 25 (1964) 5-25.

24. A. J. Peressini, Ordered topological vector spaces, Harper and Row, New York 1967.

25. R. R. Phelps, Lectures on Choquet's Theorem, Van Nostrand, Princeton, N. J. 1966.

26. A. Pietsch, Nukleare lokalkonvexe Raume, Akademie-Verlag Berlin 1965.

27. J. R. Retherford, Notes on the Dynin-Mitiagin theorem, Lecture Notes LSU. 
28. - and Ed Dubinsky, Schauder bases and Köthe sequence spaces, Trans. Amer. Math. Soc., 130 (1968), 265-280.

29. W. Woztynski, Conditional bases in non-nuclear Fréchet spaces Studia Math., 35 (1970), 77-96.

Received January 13, 1971. Work of both authors supported by NSF Grant GP20844.

The Louisiana State University, Baton Rouge

AND

UNIVERSITY OF TEL-AVIV 



\section{PACIFIC JOURNAL OF MATHEMATICS}

\section{EDITORS}

\author{
H. SAMElson \\ Stanford University \\ Stanford, California 94305 \\ C. R. HOBBY \\ University of Washington \\ Seattle, Washington 98105
}

J. DugundjI

Department of Mathematics University of Southern California Los Angeles, California 90007

RICHARD ARENS

University of California

Los Angeles, California 90024

\section{ASSOCIATE EDITORS}
E. F. BECKENBACH
B. H. NeUmanN
F. WOLE
K. YOSHIDA

\section{SUPPORTING INSTITUTIONS}

\author{
UNIVERSITY OF BRITISH COLUMBIA \\ CALIFORNIA INSTITUTE OF TECHNOLOGY \\ UNIVERSITY OF CALIFORNIA \\ MONTANA STATE UNIVERSITY \\ UNIVERSITY OF NEVADA \\ NEW MEXICO STATE UNIVERSITY \\ OREGON STATE UNIVERSITY \\ UNIVERSITY OF OREGON \\ OSAKA UNIVERSITY \\ UNIVERSITY OF SOUTHERN CALIFORNIA
}

\author{
STANFORD UNIVERSITY \\ UNIVERSITY OF TOKYO \\ UNIVERSITY OF UTAH \\ WASHINGTON STATE UNIVERSITY \\ UNIVERSITY OF WASHINGTON \\ * * * \\ AMERICAN MATHEMATICAL SOCIETY \\ CHEVRON RESEARCH CORPORATION \\ NAVAL WEAPONS CENTER
}

The Supporting Institutions listed above contribute to the cost of publication of this Journal, but they are not owners or publishers and have no responsibility for its content or policies.

Mathematical papers intended for publication in the Pacific Journal of Mathematics should be in typed form or offset-reproduced, (not dittoed), double spaced with large margins. Underline Greek letters in red, German in green, and script in blue. The first paragraph or two must be capable of being used separately as a synopsis of the entire paper. The editorial "we" must not be used in the synopsis, and items of the bibliography should not be cited there unless absolutely necessary, in which case they must be identified by author and Journal, rather than by item number. Manuscripts, in duplicate if possible, may be sent to any one of the four editors. Please classify according to the scheme of Math. Rev. Index to Vol. 39. All other communications to the editors should be addressed to the managing editor, Richard Arens, University of California, Los Angeles, California, 90024.

50 reprints are provided free for each article; additional copies may be obtained at cost in multiples of 50 .

The Pacific Journal of Mathematics is published monthly. Effective with Volume 16 the price per volume (3 numbers) is $\$ 8.00$; single issues, $\$ 3.00$. Special price for current issues to individual faculty members of supporting institutions and to individual members of the American Mathematical Society: $\$ 4.00$ per volume; single issues $\$ 1.50$. Back numbers are available.

Subscriptions, orders for back numbers, and changes of address should be sent to Pacific Journal of Mathematics, 103 Highland Boulevard, Berkeley, California, 94708.

PUBLISHED BY PACIFIC JOURNAL OF MATHEMATICS, A NON-PROFIT CORPORATION

Printed at Kokusai Bunken Insatsusha (International Academic Printing Co., Ltd.), 7-17, Fujimi 2-chome, Chiyoda-ku, Tokyo, Japan. 


\section{Pacific Journal of Mathematics}

\section{Vol. 37, No. $2 \quad$ February, 1971}

Charles Compton Alexander, Semi-developable spaces and quotient images of metric spaces .................................... 277

Ram Prakash Bambah and Alan C. Woods, On a problem of Danzer. . . . . . . . . 295

John A. Beekman and Ralph A. Kallman, Gaussian Markov expectations and related integral equations . ....................................

Frank Michael Cholewinski and Deborah Tepper Haimo, Inversion of the Hankel

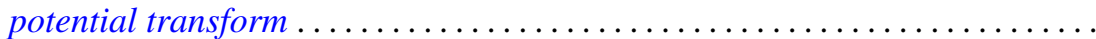

John H. E. Cohn, The diophantine equation

$$
Y(Y+1)(Y+2)(Y+3)=2 X(X+1)(X+2)(X+3) \ldots \ldots \ldots \ldots \ldots
$$

Philip C. Curtis, Jr. and Henrik Stetkaer, A factorization theorem for analytic

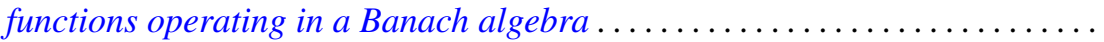

Doyle Otis Cutler and Paul F. Dubois, Generalized final rank for arbitrary limit ordinals

Keith A. Ekblaw, The functions of bounded index as a subspace of a space of entire functions

Dennis Michael Girard, The asymptotic behavior of norms of powers of

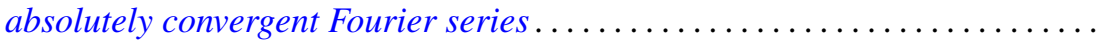

John Gregory, An approximation theory for elliptic quadratic forms on Hilbert spaces: Application to the eigenvalue problem for compact quadratic forms. 383

Paul C. Kainen, Universal coefficient theorems for generalized homology and stable cohomotopy.

Aldo Joram Lazar and James Ronald Retherford, Nuclear spaces, Schauder bases, and Choquet simplexes.

David Lowell Lovelady, Algebraic structure for a set of nonlinear integral operations

John McDonald, Compact convex sets with the equal support property . 429

Forrest Miller, Quasivector topologies

Marion Edward Moore and Arthur Steger, Some results on completability in commutative rings.

A. P. Morse, Taylor's theorem

Richard E. Phillips, Derek J. S. Robinson and James Edward Roseblade, Maximal subgroups and chief factors of certain generalized soluble groups.

Doron Ravdin, On extensions of homeomorphisms to homeomorphisms ...

John William Rosenthal, Relations not determining the structure of $\mathrm{L}$

Prem Lal Sharma, Proximity bases and subbases ........... .

Larry Smith, On ideals in $\Omega_{*}^{u}$. .

Warren R. Wogen, von Neumann algebras generated by operators similar to normal operators

R. Grant Woods, Co-absolutes of remainders of Stone-Čech 